\title{
SIMPLE NON-RADIOACTIVE METHODS OF ANALYSIS OF POLYMORPHIC MARKERS FLANKING HUMAN APOLIPOPROTEIN C-III GENE.
}

\author{
ANDREI P. SURGUCHOV, SHAO-BIN LIU AND WOLFGANG PATSCH \\ Department of Medicine, Mail Station F-701, Baylor College of Medicine, One Baylor Plaza, Houston, \\ TX 77030, USA.
}

\begin{abstract}
SUMMARY
We describe two rapid non-radioactive methods for the analysis of polymorphic markers in the flanking region of the human apolipoprotein CIII gene. The polymorphic markers comprise previously described variable sites located upstream from the coding region of the gene $\left(\mathrm{C}_{-}{ }^{641} \rightarrow \mathrm{A}, \mathrm{G}-{ }^{630} \rightarrow \mathrm{A}, \mathrm{T}-{ }^{625} \rightarrow\right.$ deletion, $\mathrm{C}_{-}{ }^{482} \rightarrow \mathrm{T}$, and T- $-455 \rightarrow \mathrm{C}$ ) and a polymorphic Sst $/$ SacI site in the apoC-III 3' untranslated region. The first method is allele-specific amplification (ASA) with primers complementary to the normal ("wild-type") allele or to the variable ("mutant") allele at their 3' ends. The other is allele-specific oligonucleotide hybridization (ASO hybridization) with pairs of probes labeled by digoxigenin. Comparison with sequencing data showed that both methods are reliable for polymorphism analysis.
\end{abstract}

KEY WORDS: Apolipoprotein C-III Polymorphism Promoter Polymerase chain reaction Genotype analysis

\section{INTRODUCTION}

Apolipoprotein C-III (apoC-III) is a major protein constituent of triglyceride-rich lipoproteins and is synthesized predominantly in the liver. In vitro studies have shown that apoC-III inhibits the hydrolysis of lipids by lipoprotein lipase (Brown and Baginsky, 1972) an enzyme participating in the clearance of triglyceride-rich lipoproteins. Several independent investigations including overexpression of apoC-III in transgenic mice demonstrated a causal role of apoC-III in the development of hypertriglyceridemia (Schonfeld et al., 1979; Malmendier et al., 1989; Ito et al., 1990). A polymorphic SstI site (S2 allele) in the apoC-III 3' untranslated region as well as several polymorphic sites in the 5 ' region are associated with elevated levels of plasma triglycerides (Rees et al., 1983; Tas, 1989; Dammerman et al., 1993). Hypertriglyceridemia (HTG) is etiologically heterogeneous and the role of apoC-III in different types of HTG requires further study. For this purpose a rapid, safe and reliable method is required to determine apoC-III polymorphic markers. Analysis of these, as well as other polymorphic markers, was previously conducted using radioactive probes. To analyze the relevance of polymorphic sites in the flanking regions of the apoC-III gene to the elevation of triglycerides, we developed two non-radioactive methods which can be used both in population and clinical studies. 


\section{MATERIALS AND METHODS}

\section{Samples for DNA isolation}

DNA was isolated from peripheral blood leukocytes of subjects with different types of lipid metabolism disorders. Genomic DNA was extracted from frozen buffy coats by the salting out procedure (Miller et al., 1988).

\section{Oligonucleotides}

Oligonucleotides (Table I and Figure 1) were synthesized using a Cyclone Plus DNA synthesizer with reagents from the Milligen Biosearch Division (Burlington, MA) and purified by thin layer chromatography. The purity of the oligonucleotide was verified by electrophoresis in a denaturing $20 \%$ polyacrylamide gel.

\section{Polymerase Chain Reaction (PCR) conditions}

Two types of PCR were performed: i) PCR of the sequence from position - 699 to -188 was carried out in order to amplify promoter fragments for subsequent analysis by ASO hybridization and sequencing. The amplification was performed in a DNA Thermal Cycler (Perkin Elmer, Cetus) according to the method of Emi and coworkers (Emi et al., 1988) using primers 1 and 2 described by Dammerman et al. ,1993 (Table 1). The size of the PCR products was determined by electrophoresis in a $10 \%$ denaturing polyacrylamide gel. ii) PCR for Allele-Specific Amplification (ASA) was conducted in order to identify variable bases both in the promoter region and in 3'-flanking untranslated region of the apoC-III gene. Two alternative primers were used to detect a particular mutation, one that is perfectly matched to the "wild type" (1/1) allele at its 3'-end ("wild-type" alternative primer), while the other was non-complementary to the "wild type" allele at 3 '-end but formed a perfect duplex with the "mutant" sequence $(2 / 2)$ (Table 1 , primers 3 - 12, Fig. 1). The conditions of PCR are described in (Postnikov et al., 1993). The following combinations of primers were used: site -625/-630/-641 — primers 3 or 4 with primer 2 ; site -482 — primers 5 or 6 with primer 9 ; site -455 — primers 7 or 8 with primer 1; SstI site - primers 10 or 11 with primer 12 .

\section{Oligonucleotide labeling for ASO-Hybridization}

Oligonucleotides 13 - 22 were labeled at the 3 ' end by incorporation of digoxigeninlabeled nucleotide and detected by immunochemiluminescence using a high-affinity sheep antidigoxigenin Fab-fragment (Boerhinger Manheim "DIG Nucleic Acid Detection Kit" and "LumiPhos ${ }^{\mathrm{TM}}$ 530"). The labeling reaction was carried out with terminal transferase and stopped by adding glycogen. Labeled oligonucleotides were precipitated by $\mathrm{LiCl} / \mathrm{ethanol}$, washed with $70 \%$ ethanol (v/v), dried under vacuum, and redissolved in distilled water (Boerhinger Manheim "DIG Nucleic Acid Detection Kit" and "LumiPhosTM 530"). Prehybridization and hybridization of digoxigenin-labeled oligonucleotides were performed as described for $\mathrm{P}^{32}$-labeled probes (Dammerman et al., 1993).

\section{Nucleotide sequence of PCR products was determined by automated Taq}

DyeDeoxyTM Terminator Cycle Sequencing (Applied Biosystems) with one unlabeled oligonucleotide as a primer. PCR products were purified by electrophoresis in $1 \%$ agarose with subsequent purification on QIAEX columns (Qiagen Inc., Chatsworth, 
Table 1.

\begin{tabular}{l}
$\underline{\text { strand localization }} \frac{\text { wt }}{\frac{\text { or }}{\text { mutant }}} \underline{\underline{\text { used for }}}$ \\
\hline
\end{tabular}

I. Primers used for PCR, Allele-Specific Amplification and Sequencing

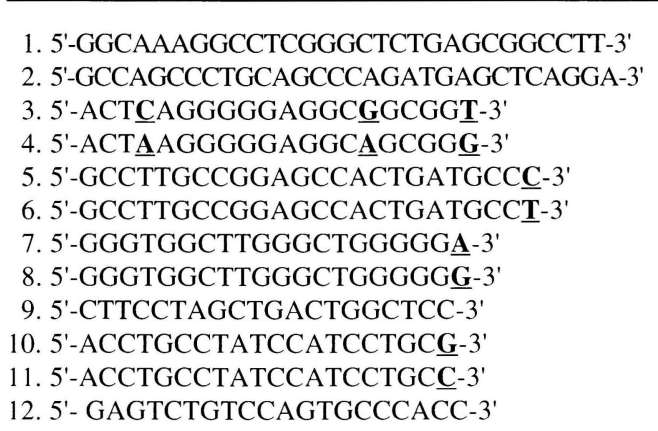

II. Probes used for ASO-hybridization (Dammerman et al., 1993)

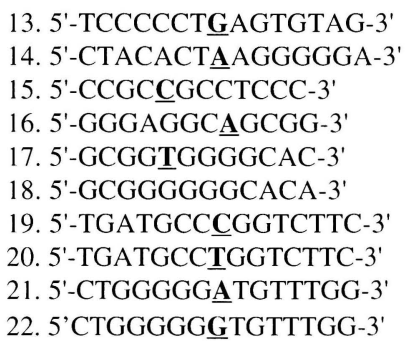

13. 5'-TCCCCCTE्GAGTGTAG-3'

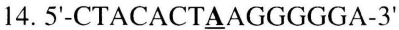

(1) 3

16. 5'-GGGAGGC스GCGG-3'

17. 5'-GCGGTGGGGCAC-3'

18. 5'-GCGGGGGGCACA-3'

19. 5'-TGATGCCCGGTCTTC-3'

21. 5'-CTGGGGGA TGTTTGG-3'

22. 5'CTGGGGGGTGTTTGG-3'

$\begin{array}{rlll}\text { S } & -699--671 & \text { wt } & \text { PCR } \\ \text { AS }-217--188 & \text { wt } & \text { PCR } \\ \text { S } & -644--625 & \text { wt } & \text { ASA }-625 /-630 /-641 \\ \text { S } & -644--625 & \mathrm{~m} & \text { ASA }-625 /-630 /-641 \\ \mathrm{~S}-482-505 & \mathrm{wt} & \text { ASA }-482 \\ \mathrm{~S}-482-505 & \mathrm{~m} & \text { ASA }-482 \\ \text { AS }-436--455 & \mathrm{wt} & \text { ASA }-455 \\ \text { AS }-436--455 & \mathrm{~m} & \text { ASA }-455 \\ \text { AS }-247--266 & \mathrm{wt} & \text { ASA }-482 \\ \mathrm{~S} & 3156-3175^{*} & \mathrm{~m}(\mathrm{~S} 2) & \text { ASA SstI } \\ \mathrm{S} & 3156-3175^{*} & \text { wt (S1) } & \text { ASA SstI } \\ \text { AS } 3386-3405^{*} & \text { wt } & \text { ASA SstI }\end{array}$

AS -634- -648 wt -641

$\mathrm{S}-634--648 \mathrm{~m} \quad-641$

AS $-632--644$ wt -630

$\mathrm{S}-632--644 \mathrm{~m} \quad-630$

S $-629--618 w t \quad-625$

S $-629--617 \mathrm{~m} \quad-625$

S $-489--475$ wt -482

S $-489--475 \mathrm{~m} \quad-482$

AS $-448--462$ wt -455

AS $-448--462 m \quad-455$

*numbering of basis in 3' -untranslated region is given as in (Protter et al., 1984), in 5'- promoter region according to (Dammerman et al., 1993). Variable bases are shown in bold and underlined.

CA). The sequencing reaction was carried out in a thermal cycler with purified PCR product (150-250 ng) as template, one of the primers (sense or antisense, 1 pmole) and dideoxynucleotides labeled with different fluorescent dyes under conditions of linear amplification of the reaction product as recommended by the manufacturer (Applied Biosystems. TaqDyeDeoxy ${ }^{\text {TM }}$ Terminator Cycle Sequencing Kit, Part \#901497).

\section{RESULTS AND DISCUSSION}

Identification of polymorphic genotype markers by ASO-hybridization

When PCR-products, obtained from the amplification of genomic DNA with primers 1 and 2, were hybridized with either "wild type" or "mutant" (see Table I, section II) digoxigenin-labeled probes one of the following patterns was observed: i) One of the alternative probes ("wild type" or "mutant"; definition of "wild type" and "mutant" is described in Dammerman et al., 1993) gave an intense signal (Fig. 2, 1a and 3b) while the other one produced little or no signal (Fig. 2, 1b and 3a). These samples were 


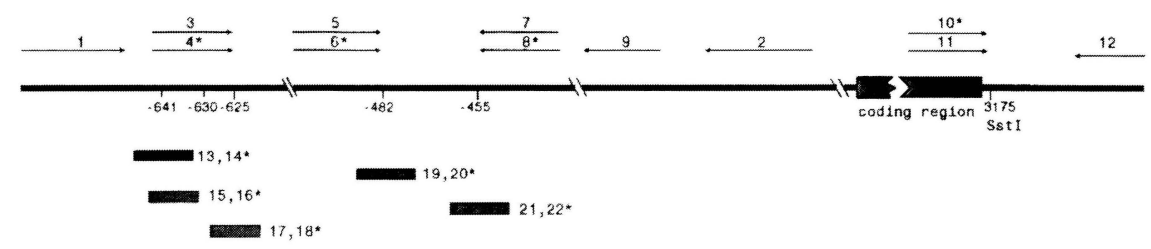

Figure 1. Localization of primers for PCR and probes for ASO-hybridization on apoC-III gene map. * primer or probe specific to a "mutant" sequence.

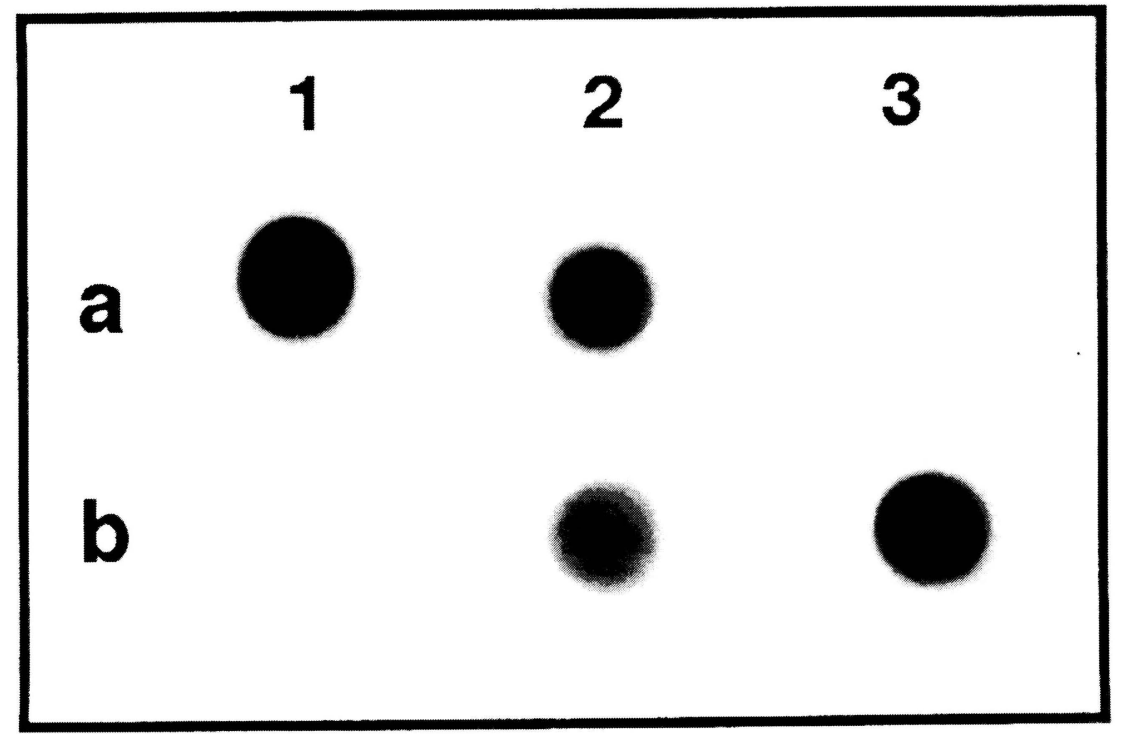

Figure 2. Results of genotyping of -482 marker by ASO-hybridization with digoxigenin labeled probes. Hybridization with probe 272 specific to "wild type" sequence (a) and with probe 273 specific to "mutant" sequence (b). DNA isolated from individual with $1 / 1$ genotype for this marker (1), 1/2 genotype (2) and $2 / 2$ genotype (3). 
a)

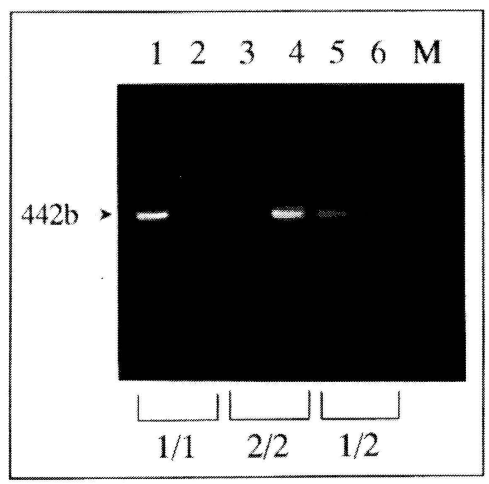

c)

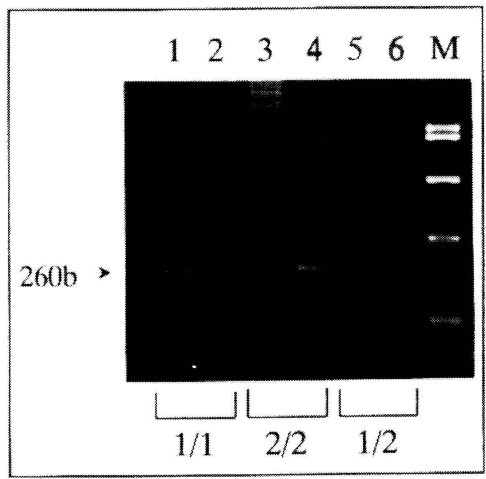

b)

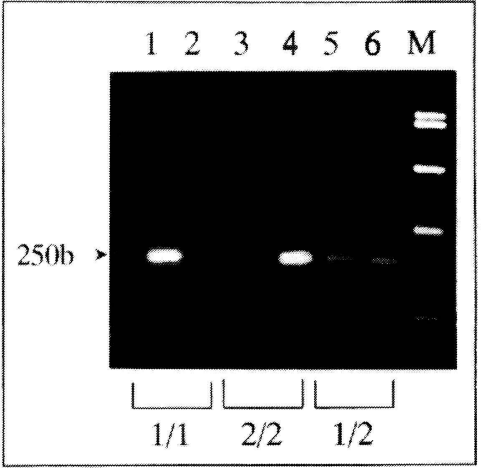

d)

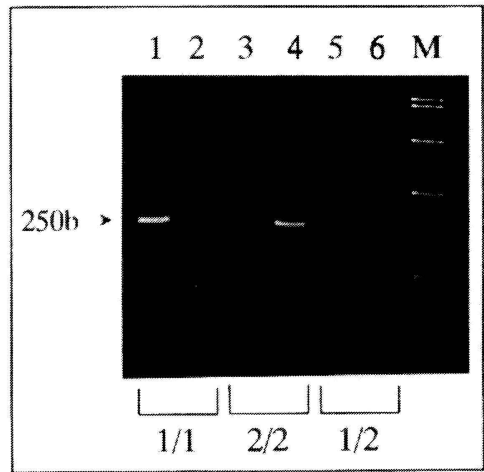

Figure 3. Results of genotyping of $-641 /-630 /-625$ (a), -482 (b), -455 (c) and SstI (d) markers by ASA. DNA was isolated from individuals with $1 / 1,1 / 2$ and $2 / 2$ genotype and used for PCR with pairs of primers obtained for the corresponding marker. 1,3 and 5-PCR with "wild type" alternative primer; 2, 4 and 6-with "mutant" alternative primer. Right lane is $1 \mathrm{~kb}$ ladder.

considered homozygous for the marker ( $1 / 1$ or $2 / 2$ depending on which of the two probes gave a signal). ii) Both of the probes gave a signal and the intensity of each signal was roughly half of the intensity observed in homozygous samples (Fig. 2, 2a and 2b). These samples were considered heterozygous (1/2) for the marker which was analyzed. Hybridization results for other markers looked similar (not shown).

The classification of the genotypes for all five markers studied (-641, -630, -625, -482 and -455) was highly reliable and the sequences of PCR products used for both ASO and ASA were confirmed by automated sequencing.

\section{Identification of polymorphic markers by ASA}

The results of ASA for different markers are presented in Fig. 3. Since the genotype at site -625 predicts, in the majority of cases, the genotypes at sites -641 and -630 (Dammerman et al., 1993 and our own preliminary results based on ASO-hybridization) 
we have used a pair of primers $3 / 4$ containing all three variable bases either in the "wild type" (primer 3) or "mutant" form (primer 4). In addition to the variable markers analyzed by ASO-hybridization we used primers capable of differentiating the SstI polymorphic marker located in the 3'-untranslated region.

Forty DNA samples typed by ASO-hybridization were subsequently analyzed by ASA and for all of them the initial typing was confirmed. Furthermore, the sequence analysis of nine DNA samples (three DNA samples for each genotype, i.e. 1/1, 1/2 and 2/2) also confirmed the results obtained by ASO-hybridization and ASA.

\section{Comparison of two methods}

Each of the two methods described for polymorphism analysis has some advantages and disadvantages. While ASO-hybridization is more time-consuming at preliminary stages (e.g., probe labeling, purification of PCR-products, etc.) it is convenient at the last step because many samples can be analyzed simultaneously by the dot-blot procedure. The method is especially convenient for the analysis of multiple markers located in close proximity (usually within $1 \mathrm{~kb}$ distance) on one chromosome since the same PCR product can be used for such analysis. Hence, ASO-hybridization may be superior for epidemiological studies when a large number of samples can be analyzed at the same time and/or for the analysis of closely located multiple markers.

The main advantage of ASA is that it is a one step direct and quick method that allows genotyping using genomic DNA. However, sometimes it is difficult to eliminate nonspecific extra bands which may appear in PCR products in addition to the main band (see for example Fig. 3c, lanes 2 and 3). Though these extra bands usually do not prevent the correct genotyping it is desirable to eliminate them or reduce their relative amount in order to increase the reliability of results.

\section{REFERENCES}

Brown, W.V., Baginsky, M.L. (1972). Inhibition of lipoprotein lipase by an apolipoprotein of human very low density lipoproteins. Biochem. Biophys. Res. Commun., 46, 375-382.

Dammerman, M.D., Sandkuijl, L.A., Halas, J.L., Chung, W., Breslow, J.L. (1993). An apolipoprotein CIII haplotype protective against hypertriglyceridemia is specified by promoter and 3' untranslated region polymorphisms. Proc. Natl. Acad. Sci. USA, 90, 4562-4566.

Emi, M., Wu, L.L., Robertson, M.A., Myers, R.L., Hegele, R.A., Williams, R.R., White, R. and Lalouel, J.-M. (1988). Genotyping and sequence analysis of apolipoprotein E isoforms. Genomics, 3, 373-379.

Ito, Y., Azrolan, N., O'Connell, A., Walsh, A., Breslow, J.L. (1990). Hypertriglyceridemia as a result of human apo CIII gene expression in transgenic mice. Science, 249, 790-793.

Malmendier, C.L., Lontie, J.F., Delcroix, C., Dubois, D.Y., Magot, T., DeRoy, L. (1989). Apolipoproteins C-II and C-III metabolism in hypertriglyceridemic patients. Effect on a drastic triglyceride reduction by combination diet restriction and fenofibrate administration. Atherosclerosis, 77, 139-149.

Miller S.A., Dykes D.D., Polesky H.F.. (1988). A simple salting out procedure for extracting DNA from human nucleated cells. Nucleic Acids Res, 16, 1215.

Postnikov, Yu.V., Molchanova, T.P., Huisman, T.J.H. (1993). Allele-specific amplification for the identification of several hemoglobin variants. Hemoglobin, 17, 439-452.

Protter, A.A., Levy-Wilson, B., Miller, J., Bencen, G., White, T., Seilhamer, J. (1984). Isolation and sequence analysis of the human apolipoprotein CIII gene and the intergenic region between the Apo AI and Apo CIII genes. DNA, 3, 449-457. 
Quarfordt, S.H., Michalopoulos, G., Schirmer, B. (1982). The effect of human C apolipoproteins on the in vitro hepatic metabolism of triglyceride emulsions in the rat. J. Biol. Chem., 257, 14642-14647.

Rees, A., Shoulders, C.C., Stocks, J., Galton, D.J., Baralle, F.E. (1983). DNA polymorphism adjacent to the human apoprotein A-I gene: Relation to hypertriglyceridemia. Lancet, 1, 444446.

Schonfeld, G., George, P.K., Miller, J., Reilly, P., Witztum, J. (1979). Apolipoprotein C-II and CIII levels in hypertriglyceridemia. Metabolism, 28, 1001-1010.

Tas, S. (1989). Strong association of a single nucleotide substitution in the $3^{\circ}$-untranslated region of the apolipoprotein-CIII gene with common hypertriglyceridemia in Arabs. Clin. Chem., 35, 256-259. 


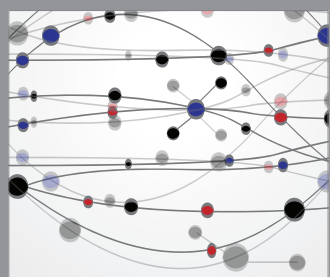

The Scientific World Journal
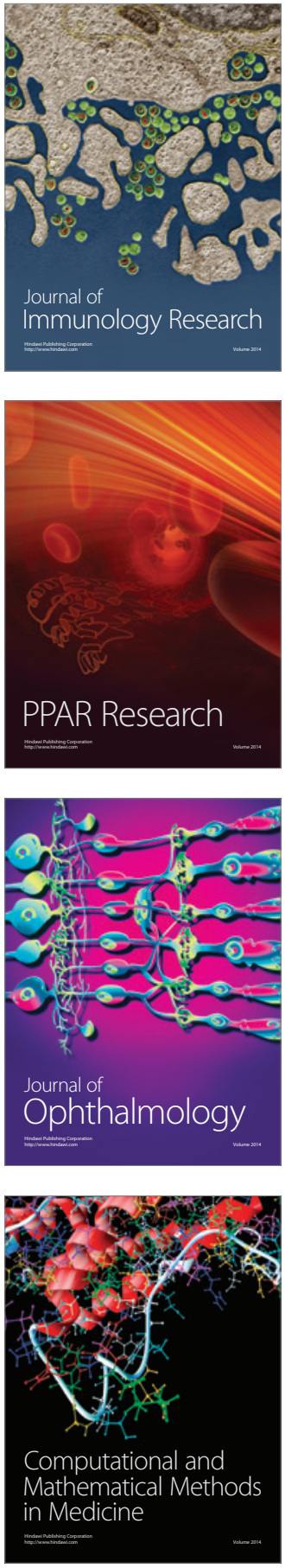

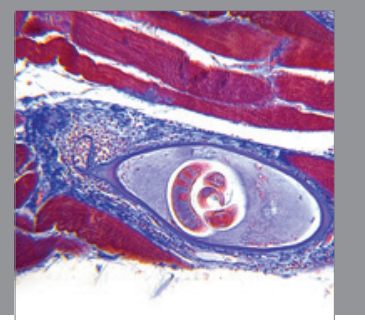

Gastroenterology

Research and Practice
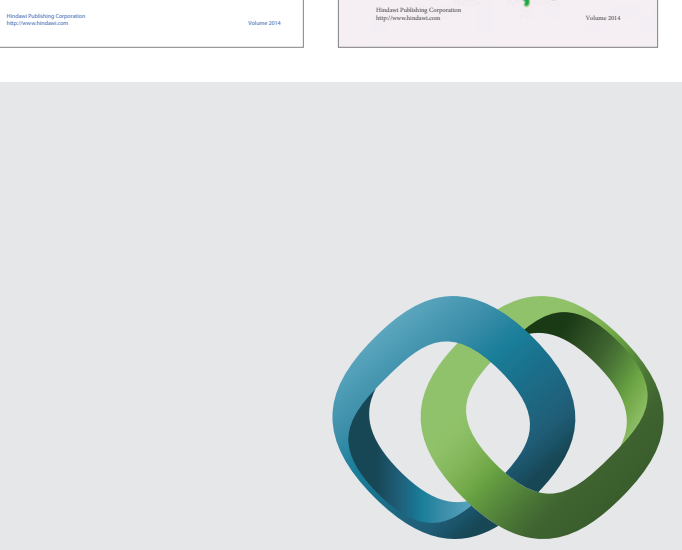

\section{Hindawi}

Submit your manuscripts at

http://www.hindawi.com
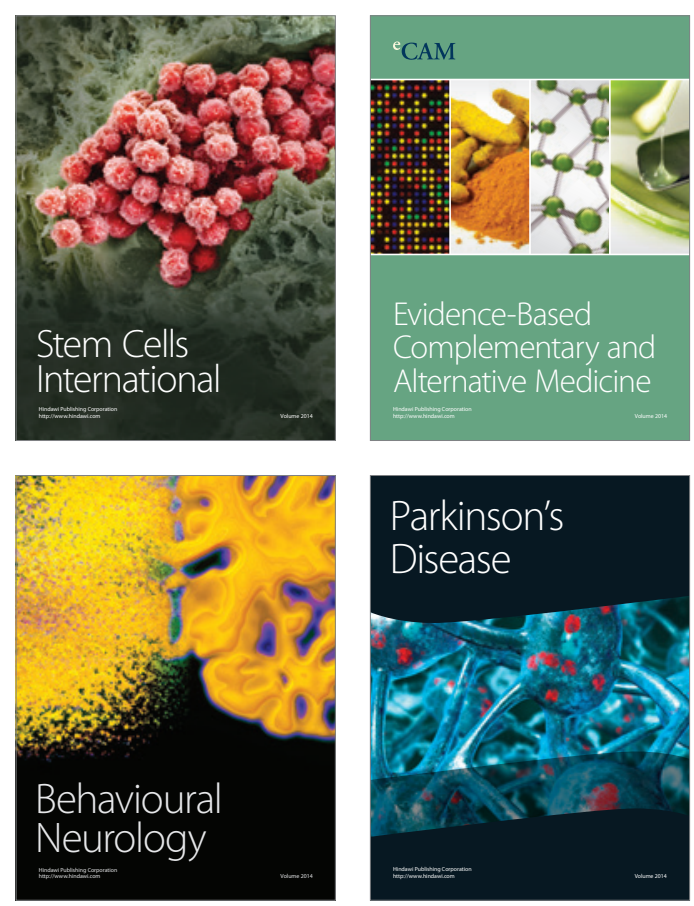

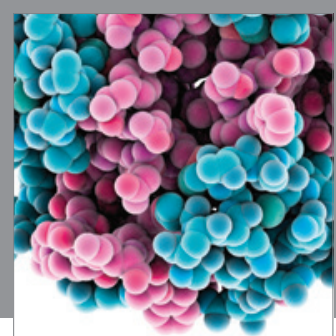

Journal of
Diabetes Research

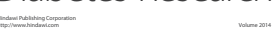

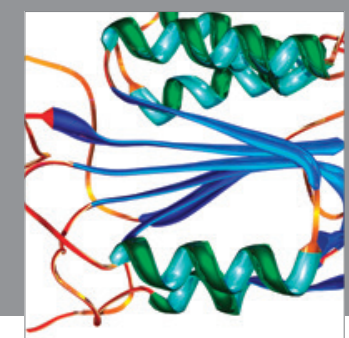

Disease Markers
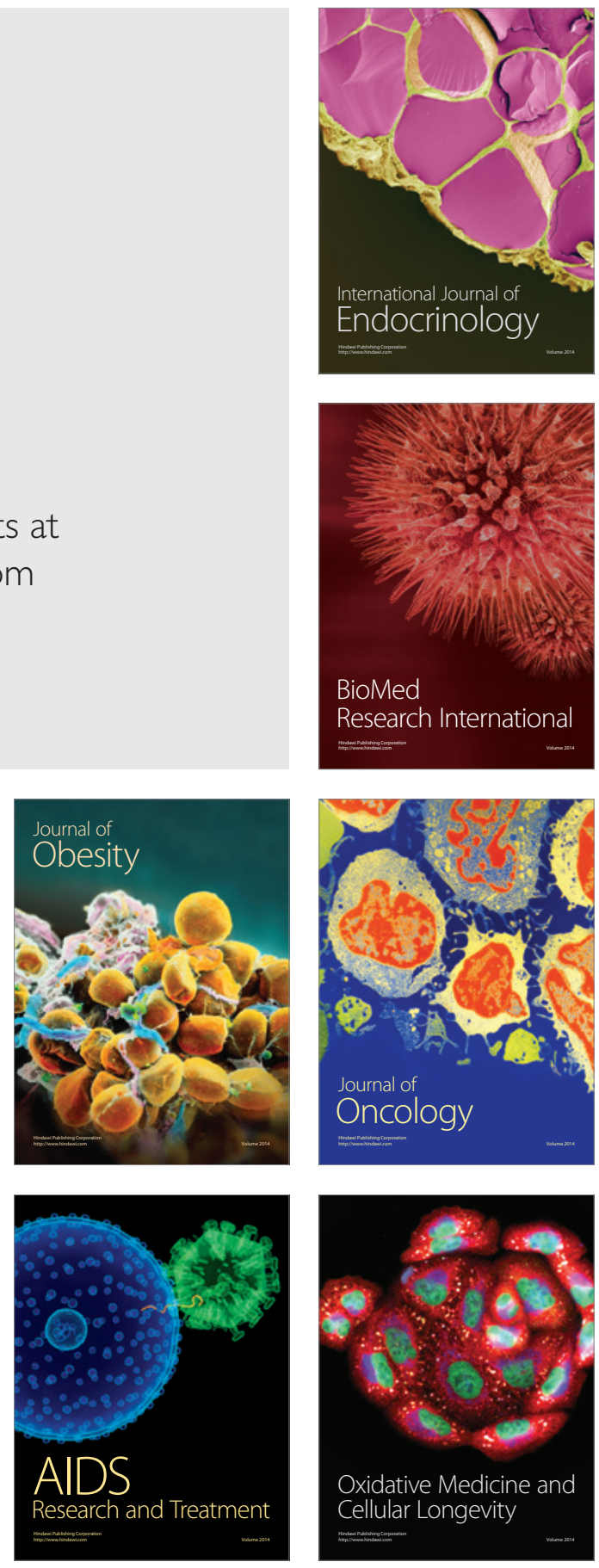